\title{
Promoción de la inversión privada y asociaciones publico privadas en la región Arequipa, como medios para la reducción de brechas de infraestructura y crecimiento económico.
}

Promotion of private investment and public-private partnerships in the Arequipa región, at means to reduce infrastructure gaps and economic growth.

Gambetta Paredes, Brunno Alonso

Universidad Católica de Santa María. Arequipa Perú.

\section{INFORMACIÓN}

\section{Historia del Artículo}

Recepción: 12/04/2019

Revisión: 16/06/2019

Aceptación: 18/06/2019

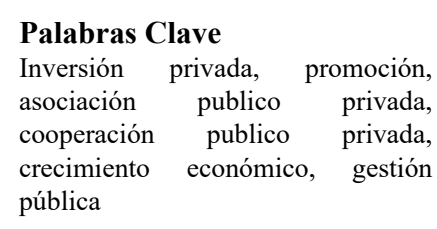

\section{Key Words}

Private invetment, promotion, private public partnership, public private cooperation, economic growth, public administration.

\section{DOI}

https://doi.org/10.35286/veritas. v20i1.225

\begin{abstract}
RESUMEN
Este articulo tiene por objetivo dar a conocer las implicancias referidas a la brecha de infraestructura por la que viene atravesando nuestra economía, la misma que viene afectando a diversos sectores productivos y al crecimiento económico de la región Arequipa y del país en su conjunto. Así mismo, se busca demostrar cómo ello puede ser paliado a través de la promoción de inversión privada y el desarrollo de Asociaciones Publico Privadas (APPs), las cuales deben ser llevadas a cabo tanto a nivel del gobierno nacional, a través de Proinversión y el Ministerio de Economia y Finanzas; y lo propio, a través de los gobiernos regionales y locales, mediante sus oficinas de promoción de la inversión privada (OPIP's). Todo lo anterior se encuentra enmarcado en la "Ley de Promoción de la Inversión Privada", la cual entró en vigencia en el año 2008, mediante el Decreto Legislativo $\mathrm{N}^{\circ} 1012$, el mismo que ha venido adquiriendo especial importancia, debido a la coyuntura actual que experimenta nuestra economía, caracterizada por gobiernos subnacionales que en los últimos años, han venido percibiendo menores recursos públicos para la inversión en proyectos de infraestructura pública, limitando su capacidad de atender la problemática antes descrita. Finalmente, se detallan algunas acciones específicas que ha venido llevando a cabo la Gerencia Regional de Promoción de la Inversión Privada del Gobierno Regional de Arequipa en esta materia, desde su creación hasta la actualidad, con el objetivo de contribuir a la reducción de las brechas en infraestructura y sobre todo al crecimiento económico nacional.
\end{abstract}

\begin{abstract}
The objective of this article is to make known the implications related to the infrastructure gap that our economy has been going through, the same which has affected various productive sectors and the economic growth of the Arequipa región and the country as a whole. Likewise, the article seeks to demonstrate how this can be alleviated through the promotion of private investment and the development of Public Private Partnerships (PPPs), which must be carried out both at the national government level, through Proinversion and the Ministry of Economy and Finance, and the same, through regional and local governments, through their offices for the promotion of private investment (OPIPs in Spanish). All of the above is framed in the "Law for the Promotion of Private Investment", which came into force in 2008, through Legislative Decree No. 1012, which has been acquiring special importance, due to the current situation that our economy experiences, characterized by subnational governments that in recent years have been receiving less public resources for investment in public infrastructure projects, limiting their capacity to address the problems described above. Finally, some specific actions that the Regional Department for the Promotion of Private Investment of the Regional Government of Arequipa has been carrying out in this area, from its creation to the present, are detailed.
\end{abstract}

\section{INTRODUCCIÓN}

En un escenario socioeconómico y político actual, donde las expectativas por parte de los agentes económicos por un mayor desarrollo y bienestar son importantes, una de las principales acciones y desafíos permanentes del estado que ha venido persiguiendo dicho cometido, pero que lamentablemente, a la fecha ha obtenido resultados incipientes, está referida a la reducción de brechas de infraestructura pública en los principales sectores de la economía nacional.

Correspondencia:

Brunno Gambetta Paredes.

brunnogambetta@gmail.com
En línea de lo anterior, es importante señalar que en los últimos años, el Gobierno Regional de Arequipa, ha venido experimentando una caída sustancial de los recursos económicos disponibles para la inversión en obras de infraestructura pública, básicamente por una reducción del canon. Sumado a ello, el desconocimiento de los inversionistas acerca de los proyectos de inversión prioritarios en la región; las modalidades de participación de la inversión privada, y la corta visión compartida sobre las necesidades de infraestructura en la región, han dificultado que se haya podido reducir las brechas de infraestructura pública en sectores estratégicos como transporte, salud, educación, energía, etc., lo cual se traduce en importantes costos sociales, en términos de acceso a servicios del estado y perdida de la competitividad. 


\section{Importancia de la Infraestructura para la competitividad}

Es importante reconocer que en el mundo en que vivimos, las economías y países que experimentan el mayor desarrollo son aquellos que han logrado construir en el tiempo esquemas de incremento de la competitividad. Así mismo, instituciones como el Foro Económico Mundial, líder en establecer el ranking de competitividad mundial, ha determinado que uno de los pilares mas importantes es la generación de la infraestructura; lo cual tiene bastante asidero, en el sentido de que las economías que presentan las mayores brechas de infraestructura enfrentan diversos costos en términos logísticos, de eficiencia, acceso a servicios, productividad, etc.

En el ámbito nacional, Serven y Calderón (2004), señalan que el desarrollo de la infraestructura tiene un impacto positivo sobre el crecimiento económico y la distribución del ingreso. En el caso peruano, si se desarrollara la infraestructura social y productiva a niveles similares a los alcanzados por Costa Rica, el PBI se incrementaría en un 3,5\% anual y en un 2,2\% si se lograran los niveles de Chile.

Así también, Vásquez y Bendezú (2008), señalan que si la infraestructura vial creciera un $1 \%$ el PBI aumentaría un $0,218 \%$, debido a que ello favorece la incorporación de nuevos sectores productivos. Por tanto, se considera que existe una relación directa entre inversión, competitividad y crecimiento del PBI. Los países y regiones con mayores niveles de inversión son los más competitivos y, por ello, tienen mayores niveles de inversión y, en consecuencia, un mayor crecimiento económico.

Con relación al déficit de infraestructura, según el Instituto Peruano de Economía IPE (2009), la brecha a nivel nacional se estima en US\$37 760 millones para el año 2018, lo que representa el $30 \%$ del PBI. De este total, los sectores transporte y energía representan en conjunto necesidades de inversión que superan los dos tercios.

Y finalmente, es de particular preocupación en el Perú, que en el marco del cierre de brechas de infraestructura, durante los últimos años la economía nacional ha venido obteniendo resultados favorables en términos de reducción de la pobreza y crecimiento económico; lo cual conlleva a una mayor demanda de infraestructura pública de parte de los peruanos. En este sentido por mas que se hayan venido ejecutando diferentes proyectos de infraestructura en diversos sectores de la economía, la demanda por los mismo también ha venido en aumento; lo cual podría estar ocasionando que las brechas de infraestructura en el país hayan venido creciendo o al menos reduciéndose en un nivel inferior al esperado en los últimos años.

\section{Tamaño de la Brecha de Infraestructura}

Para toda economía que busca el incremento de la competitividad y crecimiento económico de largo plazo, es fundamental poder saber a ciencia cierta el tamaño de las brechas de infraestructura y los niveles de inversión requeridos para atender esta problemática.

Respecto al tamaño respecto a la brecha de infraestructura en el Perú, AFIN (2015), determinó que para el periodo 20162025, la misma alcanza un total de US\$ 159,549 millones; así mismo ha estimado que para lograr el cierre de dicha brecha, el país tendría que realizar una inversión promedio anual del 8.27\% del PBI (es decir, US\$ 15,955 millones anual).

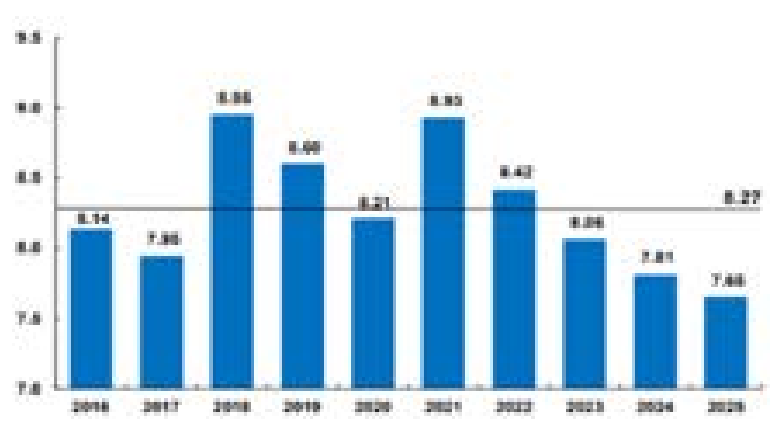

Fig.1: Costo del cierre de la brecha de infraestructura en el Perú 2016-2025. (\% del PIB). Fuente: Plan Nacional de Infraestructura 2016-2025 - AFIN

\section{Reducción presupuestal del Gobierno Regional de} Arequipa

El Gobierno Regional de Arequipa en el año 2012 registró el mayor ingreso por canon minero registrado en el periodo 2008 al 2016, teniendo un monto de S/. 195 MM. En los siguientes años los ingresos disminuyeron de manera constante registrando el monto más bajo el año 2016 el monto de S/. 5MM, según grafico siguiente:

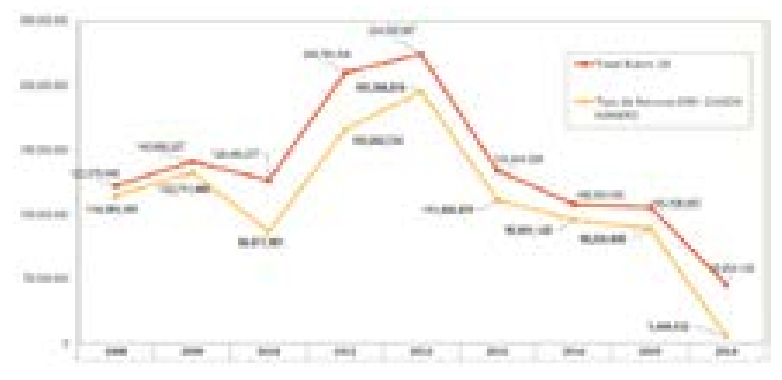

Fig.2: Transferencias recibidas por el Gobierno Regional de Arequipa por transferencias por Canon Minero 2008-2016 (En millones de soles). Fuente: Ministerio de Economía y Finanzas. Elaboración Propia.

Así mismo, se debe considerar también el endeudamiento del Gobierno Regional de Arequipa contraído por bonos soberanos, deuda pública interna (Banco Continental), y pago de CIPRL por el Proyecto "Puente Chilina"; lo que agudiza las restricciones presupuestales para ejecutar nuevos proyectos.

Tabla 1: Nivel de deuda contraída por el Gobierno Regional de Arequipa 2008-2016 (En soles). Fuente: Ministerio de Economía y Finanzas. Elaboración Propia

\begin{tabular}{ccccc}
\hline & & & & \\
Año & CIPRL & $\begin{array}{c}\text { Bonos } \\
\text { Soberanos }\end{array}$ & $\begin{array}{c}\text { Deuda Pública } \\
\text { Interna (Banco } \\
\text { Continental) }\end{array}$ & Total Deuda \\
\hline 2015 & $63,130,750$ & $11,249,690$ & & $74,380,440$ \\
2016 & $21,0.10,643$ & $10,811,790$ & $1,719,450$ & $33,541,883$ \\
2017 & $24,959,755$ & $4,374,645$ & $1,719,450$ & $31,053,850$ \\
Total & $109,101,148$ & $26,436,125$ & $3,438,900$ & $138,976,173$ \\
\hline
\end{tabular}




\section{Consecuencias de la Reducción Presupuestal}

El problema de reducción sustancial del presupuesto público y alto endeudamiento, limita enormemente la capacidad de inversión pública en la región, para entender ello, de acuerdo al siguiente cuadro; en el año 2012 con el presupuesto público disponible se hubiera podido construir 65 colegios, sin embargo, para el año 2016, solo se hubieran logrado construir 2 colegios. Esto denota de manera objetiva la drástica caída en la disponibilidad de presupuesto publico del Gobierno Regional de Arequipa, lo cual limita sustancialmente la capacidad de este ultimo para invertir en la generación de infraestructura publica demandada por la ciudadanía.

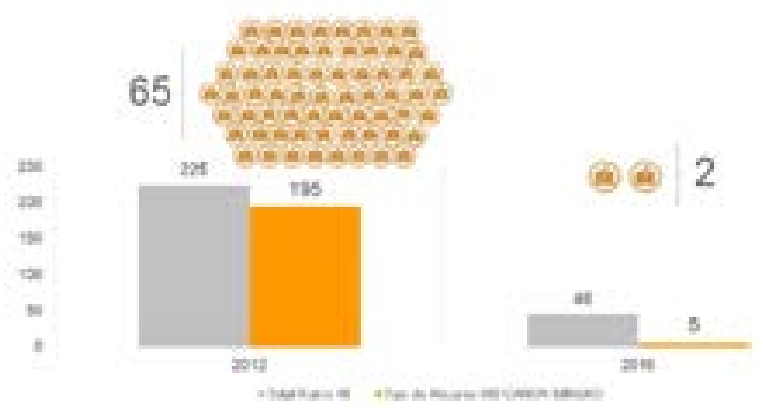

Fig.3: Evolucion del Canon Minero 2012-2016 (En millones de soles). Fuente: Gobierno Regional de Arequipa (Elaboración propia).

\section{Asociaciones Publico Privadas (APP's) y promoción de la inversión privada}

Las Asociaciones Público-Privadas (APP's), son mecanismos mediante los cuales se busca incorporar la participación articulada de los sectores público y privado, con el objetivo de estructurar, ejecutar y operar conjuntamente, proyectos de inversión en infraestructura pública, nueva o preexistente; que tradicionalmente fueron suministrados por el sector público.

En tal sentido, las Asociaciones Publico Privadas, buscan compartir las responsabilidades y riesgos derivados de la construcción y operación de la infraestructura pública resultante de manera óptima, de tal forma que se pueda minimizar el costo total, y más aún, garantizar la prestación del servicio con un alto nivel de calidad y una cobertura de largo plazo.

Myriam Ruiz Tibana y Tito Duarte (2005), sostienen que para alcanzar un desarrollo armónico es necesario establecer una interrelación vertical entre planes, programas y proyectos, todo enmarcado dentro de un proceso administrativo eficiente y visiblemente articulado entre lo público y lo privado, en ese sentido el nivel de bienestar de la sociedad, depende de la satisfacción de sus necesidades y expectativas y la solución de sus problemas, lo cual se logra a través de la eficiencia y eficacia del estado en la implementación de los planes, programas y proyectos y cooperación con los privados. Ello refuerza la necesidad de generar alianzas entre el estado y los privados para poder hacer frente a los grandes retos económicos de un territorio; y particularmente en nuestro país donde se tiene como una de las principales prioridades, la reducción de las brechas de infraestructura, lo cual esta refrendado en al Plan Nacional de Infraestructura 2016 - 2025, realizado por la Asociación de Fomento de la Infraestructura Nacional AFIN.
Así mismo, Lienhard (2006), subraya que existen ventajas en la implementación de las APP, pero también ciertos peligros, sobre todo para las autoridades públicas ya que, aunque existe una responsabilidad cedida al privado, los costos políticos siempre recaen en ellas. Por otro lado, Peters y Pierre (2010) platean que las APP's epitomizan quizás de mejor forma que otras estructuras la noción contemporánea de superioridad del mercado, en términos de eficiencia y satisfacción al cliente", por lo que añaden que estos dispositivos son cada vez diseñados menos desde el punto de vista de lo público y más como una invasión del mercado en lo público.

Si lugar a duda, las Asociaciones Publico Privadas APP's son mecanismos que le permiten al estado promover la inversión privada a todo nivel, pero que al mismo tiempo son sumamente complejos en lo técnico y operativo, los cuales requieren dentro de su estructuración, la opinión vinculante de una gran diversidad de actores, sobre todo, en el ámbito público, lo cual genera que dichos mecanismos sean ciertamente vulnerables a esquemas de corrupción. Sin embargo, es innegable afirmar que sin ellos no seria posible que se puedan gestar los megaproyectos de infraestructura que nuestra economía requiere, los mismos que demandan elevados montos de inversión y altos niveles de especialización técnica, atributos que, por su naturaleza, sobrepasan al estado, quien, amparado en una APP, logra incorporar a un socio privado que logra sortear tales exigencias.

\section{Identificación de principales trabas para la promoción de la inversión privada en la región Arequipa}

Siendo que el autor de este artículo se ha venido desempañado como Gerente Regional de Promoción de la Inversión Privada del Gobierno Regional de Arequipa, desde el periodo 2015 hasta la actualidad; responsable de atraer inversiones del sector privado en beneficio de la generación de proyectos de infraestructura publica en la región, principalmente a través de la aplicación del mecanismo de Asociaciones Publico Privadas APP's. En este marco una de las principales tareas realizadas ha sido la identificación de diferentes trabas existentes en la región Arequipa, las mismas que han venido mermando los esfuerzos llevados a cabo por el Gobierno Regional de Arequipa en esta materia, cuya identificación que se detalla a continuación, ha sido de vital importancia para definir las acciones tendientes a resolver esta problemática.

i. Reducción sustancial de los recursos públicos en el Gobierno Regional de Arequipa para ejecutar proyectos de inversión de gran envergadura:

De acuerdo Sociedad Nacional de Minería Petróleo y Energía, el Canon es un fiel reflejo de la rentabilidad del sector, en momentos de auge se captan mayores recursos, pero en periodos de crisis o reducción de precios internacionales estos serán menores. Considerando que en la región Arequipa el canon minero es el más importante en términos de montos recibidos, y que en los últimos años este sector ha venido experimentando una reducción de su performance en los mercados mundiales, esto ha generado que en los últimos años los montos de este concepto hayan venido en descenso. 


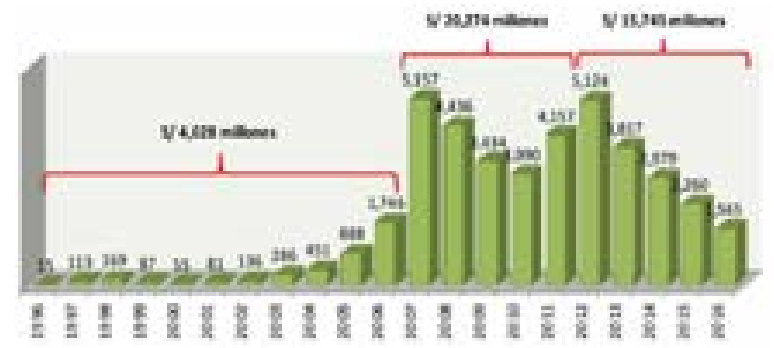

Fig.5: Transferencias de Canon Minero 1996 - 2016 (En millones de soles). Fuente: por "Sociedad Nacional de Minería Petróleo y Energía", 2016 - Reporte de Canon Minero 2016 - SNMPE.

ii. Desconocimiento del inversionista y público en general de los proyectos de inversión privada del Gobierno Regional y de las modalidades de participación del sector Privado (Asociaciones Publico Privadas)

El Gobierno Regional de Arequipa no generaba espacios ni medios de promoción de proyectos a nivel regional, ni daba a conocer al privado las distintas modalidades para ejecutar proyectos con participación privada a favor de la ciudadanía.

iii. Desconocimiento del inversionista y público en general del entorno macroeconómico de la región Arequipa $y$ del Perú, marco legal y mecanismos disponibles para la promoción de la inversión privada en el país.

No existía información consolidada de apoyo al inversionista para que conozca información económica relevante de región Arequipa, base importante para decidir invertir en la región; siendo la causa principal de esta deficiencia el desinterés y falta de proactividad por parte del sector público.

iv. Carencia de una visión compartida como institución sobre los proyectos de infraestructura estratégicos sujetos de inversión privada.

La mayoría de Gerencias Regionales son autónomas económicamente, así también en el manejo de sus recursos y proyectos, lo cual dificultaba una articulación entre todas la Gerencias para la priorización de proyectos con participación privada, identificándose como causa principal la falta de liderazgo y proactividad de parte de estas unidades orgánicas.

v. Reducido acceso por parte del inversionista a la información sobre los proyectos de inversión privada.

Si un inversionista quería invertir en Arequipa tenía que acercarse al Gobierno Regional de Arequipa y solicitar proyectos a cada Gerencia Regional, generando molestia y abandono de muchos proyectos por falta de respuesta de la entidad. La dispersión de la información era la causa principal de esta limitante y la no existencia de una gerencia que articule entre los inversionistas y el Gobierno Regional de Arequipa.
Acciones realizadas para fortalecer la labor del Gobierno Regional de Arequipa en la promoción de la inversión privada

Ante las diferentes trabas identificadas en materia de promoción de la inversión privada, el Gobierno Regional de Arequipa, en el año 2015 tomo como primera medida crear por vez primera la Gerencia Regional de Promoción de la Inversión Privada; quien a su vez producto del cumplimiento de su Manual de Operación y Funciones (MOF) en los periodos siguientes logra diseñar e implementar un sistema de promoción de inversión privada para la región, el cual está dirigido al inversionista local, nacional e internacional, y como beneficiario principal a la población de la Región Arequipa y del sur del Perú.

Como primera acción realizada en el marco del sistema estructurado, se recopiló e integro todos los proyectos potenciales para ser financiados bajo las distintas modalidades de inversión público-privada; así mismo, se incluyó información estratégica para el inversionista, el entorno macroeconómico del Perú, la situación de la inversión en la región, el marco legal para la inversión privada, entre otros. Ello permitió la elaboración y lanzamiento de la primera "Cartera de Proyectos de Inversión Privada 2015-2018", cuyo objetivo es difundir la oferta de proyectos de inversión privada en la región.

Adicionalmente se desarrolló el portal web y aplicativo Smartphone para las plataformas Android y iOS, siendo que dichos instrumentos digitalizan los proyectos de inversión privada, y además permiten al inversionista ponerse en contacto en tiempo real con los funcionarios de la gerencia para atender todo requerimiento de información.

En alianza con el sector privado, se ha logrado promover activamente la cartera de proyectos, participando de eventos nacionales e internacionales destacando presentaciones en China, España, Brasil, Chile, Polonia, República de Corea, Costa Rica, obteniéndose resultados muy favorables.

También cabe destacar la distribución de más de 2,000 carteras de proyectos en idiomas español e inglés, a diversos gremios empresariales, incluyendo diversas embajadas de países estratégicos para la inversión en el Perú.

Finalmente, se ha encontrado que desde el lanzamiento del aplicativo Smartphone para las plataformas Android y iOS (2016), se han contabilizado más de 1,000 descargas; así también se ha alcanzado alrededor de 10,000 visitas al portal de la Gerencia Regional de la Inversión Privada (www. inversionarequipa.gob.pe); contando con iniciativas privadas cuyo monto de inversión superan los $\$ 970$ Millones de dólares, que están en proceso de evaluación y algunas de ellas con un contrato de Asociación Publico Privada firmado y en ejecución.

\section{Principales logros alcanzados}

La aplicación del Sistema de Promoción de la Inversión Privada, con sus distintos componentes ha logrado que las inversiones en la región, se incrementen como se detalla a continuación:

Las cinco iniciativas privadas declaradas de interés por el Gobierno Regional, en un lapso de 06 años dentro del periodo de los años 2008 y 2014, sumaron un total de S/ 323'539,500.00; dentro de lo cual, solo un proyecto del sector Agroindustrial logró la adjudicación en el tiempo 
establecido por la norma que regula la materia, con un monto de S/. 505,000.00.

Tabla 2: Monto por Iniciativas Privadas declaradas de interés por el Gobierno Regional de Arequipa entre los años 2008 y 2014 (En soles). Fuente: Elaboración propia

\begin{tabular}{cc}
\hline Proyectos & Montos de Inversión \\
\hline Fábrica de Cemento & S/. 283,913,554.00 \\
Proyecto Agroindustrial & S/. 505,000.00 \\
Planta de Ladrillos & S/. 19,604,946.00 \\
Proyecto Hidro energético & S/. 5,183,000.00 \\
Construcción y operación del proyecto \\
complejo portuario Corío Playuelas
\end{tabular}

Por otro lado, desde la Implementación del nuevo sistema estratégico de Promoción de la Inversión Privada, la Gerencia de Inversión Privada del Gobierno Regional de Arequipa (OPIP) del Gobierno Regional de Arequipa, en el lapso de 02 años, luego de haber lanzado por vez primera la "Cartera de Proyectos de Inversión Privada", posteriormente el portal de Inversiones, y el Aplicativo Móvil, logró incrementar de manera significativa las iniciativas privadas ingresadas por montos importantes tales como:

Tabla 3: Monto por Iniciativas Privadas declaradas de interés por el Gobierno Regional de Arequipa entre los años 2015 y 2017 (En soles). Fuente: Elaboración propia

\begin{tabular}{cc}
\hline Proyectos & Monto de Inversión \\
\hline Proyecto de Infraestructura vial & $\$ 216,000,000.00$ \\
Proyecto de Residuos Solidos & $\$ 1,000,000.00$ \\
Proyecto Hidro energético & $\$ 970,000,000.00$ \\
Proyecto en Obras por Impuestos & $\$ 9,000,000,00$ \\
\hline Monto total en US\$ & $\$ 1,196,000,000.00$ \\
Monto total en S/. (Tipo de cambio en & S/. 3,946,800,000.00* \\
S/.3.3 según BCR) &
\end{tabular}

\section{CONCLUSIONES}

En los últimos años, tanto los gobiernos regionales como los locales han venido percibiendo menores recursos transferidos por el gobierno nacional provenientes principalmente por el canon y sobre canon, lo cual ha reducido las capacidades de inversión pública y generación de infraestructura.

Al mismo tiempo, en la última década, la inversión privada ha demostrado ser el motor del crecimiento de nuestra economía, superando notoriamente los niveles de inversión pública y representando los mayores aportes al crecimiento del Producto Bruto Interno del país; así mismo por su propia naturaleza es predecible que la inversión privada sea aquella que experimenta los mayores niveles de eficiencia, capacidad técnica, financiera, gestión de riesgos, entre otros.

Nuestro país se ha venido desempeñando como uno de los pioneros en la región en cuanto a la promoción de la inversión privada, a través de la ejecución Asociaciones Publico Privadas, las cuales han demostrado ser mecanismos eficientes de participación conjunta de los sectores público y privado.

El Sistema de Promoción de Inversión Privada estructurado, tiene un horizonte de largo plazo pues su constante actualización permitirá adaptarlo a las necesidades propias de la región en materia de proyectos orientados al necesario cierre de brechas de infraestructura de nuestra economía, buscando siempre un contexto de transparencia, competencia, subsidiariedad y valor por dinero.

Producto de ello, la institución ha recuperado su credibilidad y confiabilidad, requisitos indispensables para la generación de alianzas público-privadas; además, mejorándose la relación con la población, quienes ahora perciben que los esfuerzos de los funcionarios públicos se vienen traduciendo en resultados concretos en términos de mayor inversión y generación de infraestructura, incrementándose los niveles de ingresos, empleo, y sobre todo mayor acceso a servicios públicos de calidad, en beneficio de toda la población.

\section{AGRADECIMIENTOS}

A la Universidad Católica de Santa María por la publicación del presente artículo

\section{REFERENCIAS BIBLIOGRÁFICAS}

1. Asociación para el Fomento de la Infraestructura Nacional AFIN (2015). Un Plan para salir de la pobreza: Plan Nacional de Infraestructura 2016-2025, 13-14.

2. IPE, El reto de la infraestructura al 2018. Estudio encargado por la Asociación para el Fomento de la Infraestructura Nacional - AFIN. Lima, agosto de 2009, p. 15.

3. LIENHARD, A. (2006). Les Partenariats Public Privé (PPP) en Suisse. Expériences, Risques et Possibilities. Revue Internationale de Sciences Administratives, 587604.

4. PETERS, B. Y PIERRE, J. (2010). Public-Private Partnerships and the Democratic Deficit: Is Performance-Based Legitimacy the Answer? En M. Bexell y U. Mörth (Eds.). Democracy and PublicPrivate Partnerships in Global Governance (pp. 41-54). New York: Palgrave MacMillan.

5. RUIZ TIBANA, Miriam; DUARTE Tito, "Los Proyectos de Desarrollo", La Inversión Pública y la Inversión privada", Facultad de Ciencias de la Educación, Facultad de Ingeniería Industrial, Universidad Tecnológica de Pereira, Pereira Colombia, 2005.

6. SERVEN, Luis y César CALDERÓN, "The Effects of Infrastructure Development on Growth and Income Distribution”. Banco Central de Chile, Documentos de Trabajo $\mathrm{n}^{\circ} 270$, septiembre de 2004.

7. VÁSQUEZ, Arturo y Luis BENDEZÚ, Ensayos sobre el rol de la infraestructura vial en el crecimiento económico del Perú. Lima: Consorcio de Investigación Económica y Social y Banco Central de Reserva del Perú, septiembre 2008. 